Working Paper 91 - 24

September 1991
Departamento de Economía

Universidad Carlos III de Madrid

Calle Madrid, 126

28903 Getafe (Spain)

\title{
ON ROBUSTNESS PROPERTIES OF BOOTSTRAP APPROXIMATIONS
}

\author{
Antonio Cuevas* and Juan Romo**
}

\begin{abstract}
Bootstrap approximations to the sampling distribution can be seen as generalized statistics taking values in a space of probability measures. We first analyze qualitative robustness [in Hampel's (1971) sense] of these statistics when the initial estimators $\left\{T_{n}\right\}$ (whose distributions we want to approximate using bootstrap resampling) are obtained by restriction from a statistical functional $T$ defined for all probability distributions. Whereas continuity of $T$ turns out to be the natural condition to ensure qualitative robustness of $\left\{T_{n}\right\}$, we show that the uniform continuity of $T$ is a sufficient condition for robustness of the bootstrap. This result applies to M-estimators. Next, we study asymptotic properties of the bootstrap estimator for the influence function $T^{\prime}(F ; x)$ of $T$ at a distribution $F$ and we prove that continuous Hadamard differentiability of the operator $F \mapsto T^{\prime}(F ;$.$) with respect to F$ is a natural condition to establish the validity of bootstrap confidence bands for this estimator.
\end{abstract}

Key Words

Qualitative robustness, influence curve, bootstrap confidence bands, Hadamard differentiability.

* Antonio Cuevas, Departamento de Matemáticas, Universidad Autónoma de Madrid.

* Juan Romo, Departamento de Economía, Universidad Carlos III de Madrid. 


\title{
ON ROBUSTNESS PROPERTIES OF BOOTSTRAP APPROXIMATIONS
}

\author{
ANTONIO CUEVAS ${ }^{1}$ \\ Dpto. de Matemáticas \\ Universidad Autónoma \\ 28049 Madrid, Spain \\ and \\ JUAN ROMO \\ Dpto. de Economía \\ Universidad Carlos III de Madrid \\ 28903 Getafe(Madrid), Spain
}

\begin{abstract}
Bootstrap approximations to the sampling distribution can be seen as generalized statistics taking values in a space of probability measures. We first analyze qualitative robustness [in Hampel's (1971) sense] of these statistics when the initial estimators $\left\{T_{n}\right\}$ (whose distributions we want to approximate using bootstrap resampling) are obtained by restriction from a statistical functional $T$ defined for all probability distributions. Whereas continuity of $T$ turns out to be the natural condition to ensure qualitative robustness of $\left\{T_{n}\right\}$, we show that the uniform continuity of $T$ is a sufficient condition for robustness of the bootstrap. This result applies to M-estimators. Next, we study asymptotic properties of the bootstrap.estimator for the influence function $T^{\prime}(F ; x)$ of $T$ at a distribution $F$ and we prove that continuous Hadamard differentiability of the operator $F \mapsto T^{\prime}(F ;$.$) with respect to F$ is a natural condition to establish the validity of bootstrap confidence bands for this estimator.
\end{abstract}

Key words: Qualitative robustness, influence curve, bootstrap confidence bands, Hadamard differentiability.

\footnotetext{
${ }^{1}$ Research partially supported by DGICYT PB88-0178(Spain)
} 


\section{INTRODUCTION}

The old idea of considering the estimators as restrictions of statistical functionals, defined in the space of probability distributions, has proved to be extremely useful in mathematical statistics. We could mention three important applications: first, weak continuity of statistical functionals can be interpreted in terms of qualitative (infinitesimal) robustness of the corresponding sequence of estimators (Hampel, 1971). Second, the existence of a directional derivative [influence curve; see Hampel et al. (1987)] of the functional allows to define quantitative measures of robustness (gross-error sensitivity, local-shift sensitivity, rejection points). Finally, stronger differentiability properties (Fréchet, Hadamard, von Mises derivatives) provide a standard methodology to study asymptotic distributions [see, e.g., Fernholz (1983)].

In bootstrap theory, the use of the functional approach has been focussed on the third point: Fréchet and Hadamard derivatives have been employed in establishing asymptotic validity of the bootstrap [Parr (1985), Gill (1989), Arcones and Giné (1992)]. In turn, this paper is concerned with the first two issues mentioned above: our aim is to provide results of qualitative robustness for the bootstrap (Section 2 below), and to build bootstrap confidence bands for the influence curve.

In the sequel, $T_{n}=T_{n}\left(X_{1}, \ldots, X_{n}\right)$ will be (for all $\left.n=1,2, \ldots\right)$ an estimator taking values in $\mathcal{R}^{p}(p \geq 1)$, defined on random samples $X_{1}, \ldots, X_{n}$ from a univariate distribution. Let $\left(\mathcal{F}, d_{L}\right)$ be the space of probability measures on $\mathcal{R}$ (the real line) endowed with the Lévy metric $d_{L}$, which metrizes the weak convergence in $\mathcal{F}$ [see Huber (1981), p. 25].

We assume throughout that the sequence $\left\{T_{n}\right\}$ is generated by a functional $T: \mathcal{D} \subset \mathcal{F} \longmapsto \mathcal{R}^{p}$, in the sense that for all $n$ and for each sample $X_{1}, \ldots, X_{n}$, we have $T_{n}\left(X_{1}, \ldots, X_{n}\right)=T\left(F_{n}\right)$, where $F_{n}$ is the empirical distribution associated to $X_{1}, \ldots, X_{n}$. Many usual estimators fulfil this condition; this is the case, for instance, of M- and L-estimators which will be considered below [see Huber (1981)]. By $\mathcal{F}_{n}$ we will represent the set of empirical distributions of order $n$ in $\mathcal{F}$, that is, the set of discrete probability measures in $\mathcal{F}$ whose atoms have probabilities equal to $1 / n$ or to a multiple of $1 / n$. When convenient, some subsets $\mathcal{E}_{n} \subset \mathcal{F}_{n}$ will be identified with the corresponding set of points in $\mathcal{R}^{n}$, defined up to permutations of the coordinates. Obviously, the domain $\mathcal{D}$ of $T$ has to include $\mathcal{F}_{n}$ for all $n \in \mathcal{N}$. 
For each $F \in \mathcal{F}$, the symbol $\mathcal{L}_{n}(F)\left(\equiv \mathcal{L}_{n}(T ; F)\right.$ ) stands for the sampling distribution of the statistic $T_{n}\left(X_{1}, \ldots, X_{n}\right)$ when the sample is drawn from $F$. For simplicity, we omit the reference to $T$ when no confusion is possible.

The bootstrap methodology [see, e.g., Efron (1982) for introductory background] essentially consists in estimating $\mathcal{L}_{n}(F)$ by $\mathcal{L}_{n}\left(F_{n}\right)$; that is, the sampling distribution of $T_{n}$ under $F$ is approximated by the corresponding one under $F_{n}$. In an analogous way, a general operator $\varphi\left[\mathcal{L}_{n}(F)\right]$ could be estimated by $\varphi\left[\mathcal{L}_{n}\left(F_{n}\right)\right]$. A random sample of size $n$ drawn from the empirical distribution $F_{n}$ will be denoted by $X_{1}^{*}, \ldots, X_{n}^{*}$; the corresponding bootstrap empirical distribution is represented by $F_{n}^{*}$.

It is important to note that the bootstrap estimator $\mathcal{L}_{n}\left(F_{n}\right)$ can be viewed as a generalized statistic, defined on the sample space and taking values in the space $\mathcal{P}$ of probability measures on $\mathcal{F}\left(\mathcal{R}^{p}\right)$ ( $\equiv$ the space of probability distributions on $\left.\mathcal{R}^{p}\right)$; in what follows, $\mathcal{P}$ and $\mathcal{F}\left(\mathcal{R}^{p}\right)$ are both endowed with the Prokhorov metric (denoted by $d_{P}$ in both cases). Recall that $d_{P}$ is defined by

$$
d_{P}\left(P_{1}, P_{2}\right)=\inf \left\{\epsilon>0: P_{1}(A) \leq P_{2}\left(A^{\epsilon}\right)+\epsilon, \forall A \in \mathcal{B}\right\}
$$

where $A^{\epsilon}$ is the union of open balls of radius $\epsilon$ around points of $A$, and $\mathcal{B}$ denotes the Borel $\sigma$-algebra where the probability measures $P_{1}$ and $P_{2}$ are defined. $d_{P}$ metrizes the weak convergence in the space of probability measures defined on any general Polish space [see Huber (1981)].

By $\mathcal{L}\left[\mathcal{L}_{n}\left(F_{n}\right)\right]$, we will represent the sampling distribution of the generalized statistic $\mathcal{L}_{n}\left(F_{n}\right)$ in $\mathcal{P}$. So, $\mathcal{L}\left[\mathcal{L}_{n}\left(F_{n}\right)\right]$ is an element of the space of probability measures $\mathcal{P}$ (which is also endowed with the Prokhorov metric).

We use the metric $d_{L}$ in $\mathcal{F}$, instead of $d_{P}$, due to technical reasons that will be explained below. Anyway, this issue is not essential from a conceptual point of view. As we have indicated, both metrics define the same topology although the definition of $d_{L}$ (unlike that of $d_{P}$ ) is only meaningful on $\mathcal{F}$ and cannot be extended for probability measures on general spaces. Although we are considering throughout the standard form of the bootstrap, we believe that similar results could be obtained for the so called smoothed bootstrap, where the resampling is carried out from a smoothed version $\hat{F}_{n}$ (obtained, e.g. from a kernel estimator of the unknown density). 


\section{QUALITATIVE ROBUSTNESS OF BOOTSTRAP APPROXIMATIONS}

The concept of qualitative robustness [Hampel $(1968,1971)]$ formalizes the notion of robustness, against infinitesimal perturbations in the underlying model, for the case of parametric point estimates. The basic tool for this formalization is the concept of equicontinuity. More precisely, with the notation of Section 1, a sequence $\left\{T_{n}\right\}$ of parametric estimates is said to be qualitatively robust at $F \in \mathcal{F}$ if the sequence of transformations (from $\mathcal{F}$ to $\left.\mathcal{F}\left(\mathcal{R}^{p}\right)\right) G \mapsto \mathcal{L}_{n}(G)$ is asymptotically equicontinuous at $F$ (i.e., there exists $n_{0}$ such that $\left\{\mathcal{L}_{n}(G)\right\}_{n \geq n_{0}}$ is equicontinuous at $F$ ). There are other slightly different versions of this definition but all of them correspond to the same basic idea.

Rieder (1982) and Lambert (1982) have studied qualitative robustness in hypotheses testing. Boente, Fraiman and Yohai (1987) have considered the case of dependent observations. Cuevas (1988) has analyzed this concept in the context of abstract inference which concerns those inference problems whose sampling and/or parameter spaces are general (abstract) metric spaces. Our approach here is related to the latter perspective [see Grenander (1981)] since, as we mention above, we consider the bootstrap approximation $\mathcal{L}_{n}\left(F_{n}\right)$ as a generalized estimate taking values in $\mathcal{F}\left(\mathcal{R}^{p}\right)$. This leads in a natural way to the following

DEFINITION 1. Given a sequence $\left\{T_{n}\right\}$ of statistics generated by a statistical functional $T$, the sequence of bootstrap approximations $\left\{\mathcal{L}_{n}\left(F_{n}\right)\right\}$ is said to be qualitatively robust at $F$ when the sequence of transformations $G \mapsto \mathcal{L}\left[\mathcal{L}_{n}\left(G_{n}\right)\right]$ is asymptotically equicontinuous at $F$.

We will obtain qualitative robustness of bootstrap approximations through uniform qualitative robustness of the sequence $\left\{T_{n}\right\}$ :

DEFINITION 2. $\left\{T_{n}\right\}$ is uniformly qualitatively robust in a neighborhood $\mathcal{U}\left(F_{0}\right)$ of $F_{0}$ if there exists $n_{0} \in \mathcal{N}$ such that for all $n \geq n_{0}$ and for all $\varepsilon>0$ there exists $\delta>0$ such that, for all $F \in \mathcal{U}\left(F_{0}\right)$,

$$
d_{L}(F, G)<\delta \Rightarrow d_{P}\left(\mathcal{L}_{n}(F), \mathcal{L}_{n}(G)\right)<\varepsilon .
$$

It is known (Hampel, 1971) that if $\mathrm{T}$ is continuous at $F_{0}$ then the sequence $\left\{T_{n}\right\}$ is qualitatively robust at $F_{0}$. We show next that uniform continuity of 
$\mathrm{T}$ in a neighborhood of $F_{0}$ is enough for $\left\{T_{n}\right\}$ to be uniformly qualitatively robust.

THEOREM 1. If $T$ is uniformly continuous in a neighborhood $\mathcal{U}\left(F_{0}\right)$ of $F_{0}$ then $\left\{T_{n}\right\}$ is uniformly qualitatively robust in $\mathcal{U}\left(F_{0}\right)$.

Proof. It is enough to show that for all $\varepsilon>0$ there exists $\delta>0$ such that for all $F \in \mathcal{U}\left(F_{0}\right)$ there exists a sequence $\mathcal{E}_{n} \subset \mathcal{F}_{n}$ such that $F^{n}\left(\mathcal{E}_{n}\right)>1-\varepsilon$ and for $H_{n} \in \mathcal{E}_{n}$ and $G_{n} \in \mathcal{F}_{n}$ we have

$$
d_{L}\left(H_{n}, G_{n}\right)<\delta \Rightarrow\left|T_{n}\left(H_{n}\right)-T_{n}\left(G_{n}\right)\right|<\varepsilon .
$$

From Lemma 1 in Hampel (1971), it is straightforward to check that this implies uniform qualitative robustness.

Let $\varepsilon>0$. Since $\mathrm{T}$ is uniformly continuous in $\mathcal{U}\left(F_{0}\right)$, there exists $\delta_{0}>0$ such that for all $F \in \mathcal{U}\left(F_{0}\right)$

$$
d_{L}(F, G)<\delta_{0} \Rightarrow|T(F)-T(G)|<\frac{\varepsilon}{2} .
$$

By the uniformity in $\mathrm{F}$ of Glivenko-Cantelli convergence for the Lévy metric, there exists $n_{0} \in \mathcal{N}$ such that, for $n>n_{0}$,

$$
F^{n}\left(d_{L}\left(F_{n}, F\right)<\delta_{0}\right)>1-\varepsilon,
$$

for all $F \in \mathcal{U}\left(F_{0}\right)$. For $n>n_{0}$ and $F \in \mathcal{U}\left(F_{0}\right)$, define $\mathcal{E}_{n}=\left\{H_{n} \in \mathcal{F}_{n}\right.$ : $\left.d_{L}\left(H_{n}, F\right)<\frac{\delta_{0}}{2}\right\}$. Then, we have that $F^{n}\left(\mathcal{E}_{n}\right)>1-\varepsilon$ together with

$$
H_{n} \in \mathcal{E}_{n} \text { and } d_{L}\left(H_{n}, G_{n}\right)<\frac{\delta_{0}}{2}
$$

implies that

$$
d_{L}\left(H_{n}, F\right)<\frac{\delta_{0}}{2} \text { and } d_{L}\left(G_{n}, F\right)<\delta_{0} .
$$

So,

$$
\left|T_{n}\left(H_{n}\right)-T_{n}\left(G_{n}\right)\right| \leq\left|T_{n}\left(G_{n}\right)-T(F)\right|+\left|T_{n}\left(H_{n}\right)-T(F)\right|<\varepsilon,
$$


and the result follows.

Now, we show that uniform qualitative robustness of the sequence $\left\{T_{n}\right\}$ is enough to get qualitative robustness of $\left\{\mathcal{L}_{n}\left(F_{n}\right)\right\}$.

THEOREM 2. If $\left\{T_{n}\right\}$ is uniformly qualitatively robust in a neighborhood $\mathcal{U}\left(F_{0}\right)$ of $F_{0}$ then the sequence of bootstrap estimators of $\mathcal{L}_{n}(F)$ is qualitatively robust at $F_{0}$.

Proof. Fix $\varepsilon>0$. By the uniform robustness of $\left\{T_{n}\right\}$ in $\mathcal{U}\left(F_{0}\right)$, there exists $\delta>0$ such that $d_{L}(F, G)<\delta$ implies $d_{P}\left(\mathcal{L}_{n}(F), \mathcal{L}(G)\right)<\varepsilon$, for all $F \in \mathcal{U}\left(F_{0}\right)$ and for all $n$ larger than some $n_{0}$. Take $\delta_{1}=\frac{\delta}{2}$. By uniformity of Glivenko-Cantelli convergence for the Lévy metric, if $d_{L}\left(F_{0}, G\right)<\delta_{1}$ then $d_{L}\left(F_{0, n}, G_{n}\right)<\delta$ almost surely for all $n$ larger than some $n_{1} \geq n_{0}$. From the uniform robustness of $\left\{T_{n}\right\}$ it follows that $d_{P}\left(\mathcal{L}_{n}\left(F_{0, n}\right), \mathcal{L}_{n}\left(G_{n}\right)\right)<$ $\varepsilon$ (in $\mathcal{F}\left(\mathcal{R}^{p}\right)$ ) almost surely for all $n \geq n_{1}$. So, by Strassen's theorem, $d_{P}\left(\mathcal{L}\left(\mathcal{L}_{n}\left(F_{0, n}\right)\right), \mathcal{L}\left(\mathcal{L}_{n}\left(G_{n}\right)\right)\right)<\varepsilon$, for all $n \geq n_{1}$, and the result follows.

From both results, we get that uniform continuity of $T$ in a neighborhood $\mathcal{U}\left(F_{0}\right)$ of $F_{0}$ is a sufficient condition for qualitative robustness at $F_{0}$ of bootstrap approximations. Next, we show that this result is general enough to contain M-estimators.

Let $\psi$ be a non-decreasing function from $\mathcal{R}$ to $\mathcal{R}$, taking positive and negative values. If $F$ is a distribution function and $\lambda_{F}(t)=\int_{\mathcal{R}} \psi(x-t) d F(x)$ is well defined, let

$$
T(F)=\frac{1}{2}\left(\sup \left\{t: \lambda_{F}(t)>0\right\}+\inf \left\{t: \lambda_{F}(t)<0\right\}\right),
$$

when defined. The sequence $\left\{T_{n}\right\}$ obtained from $T$ is a sequence of $M-$ estimators of location.

THEOREM 3. If $\psi$ is bounded and $\lambda_{F}(t)=0$ has a unique solution for all $\mathrm{F}$ in a neighborhood $\mathcal{U}\left(F_{0}\right)$ then $T$ is uniformly continuous in $\mathcal{U}\left(F_{0}\right)$.

Proof. We follow the proof of Theorem 1 in Deniau et al. (1977) to show that given $\varepsilon>0$ we cannot have $|T(F)-T(G)|>\varepsilon$ and $d_{L}(F, G)<\delta$ for small enough $\delta$ and some $F \in \mathcal{U}\left(F_{0}\right)$. If $d_{L}(F, G)<\delta$, by Strassen's 
theorem, there exists a law $Q$ on $\mathcal{R}^{2}$ with marginals $F$ and $G$ such that $Q\{|x-y|<\delta\} \geq 1-\delta$. By definition of $T(F)$ and $T(G)$, for all $t>0$,

$$
0 \geq S(t)=\int_{\mathcal{R}^{2}}\left(\psi(x-T(F))-\psi(y-T(G)+t) d Q(x, y)=S_{1}+S_{2},\right.
$$

where

$$
S_{1}=\int_{\{|x-y| \leq \delta\}}(\psi(x-T(F))-\psi(y-T(G)+t) d Q(x, y)
$$

and

$$
S_{2}=\int_{\{|x-y|>\delta\}}(\psi(x-T(F))-\psi(y-T(G)+t) d Q(x, y) .
$$

Assume $T(G) \geq T(F)+\varepsilon$ and take $\delta<\varepsilon$ and $t \leq \varepsilon-\delta$. Since $|x-y| \leq \delta$ implies

$$
\psi(x-T(F))-\psi(y-T(G)+t) \geq \psi(x-T(F))-\psi(x+\varepsilon-T(G)) \geq 0,
$$

it follows that

$$
\begin{aligned}
S_{1} & \geq \int_{\{|x-y| \leq \delta\}}(\psi(x-T(F))-\psi(x+\varepsilon-T(G))) d Q= \\
& =\int_{\mathcal{R}^{2}}(\psi(x-T(F))-\psi(x+\varepsilon-T(G))) d Q- \\
& -\int_{\{|x-y|>\delta\}}(\psi(x-T(F))-\psi(x+\varepsilon-T(G))) d Q .
\end{aligned}
$$

The absolute value of the last integral above is bounded by $\delta$ sup $|\psi|$ and since $\lambda_{F}(t)=0$ has a unique solution for all $F \in \mathcal{U}\left(F_{0}\right)$, the first integral in the right hand side is strictly positive. It follows that, for small enough $\delta, \mathrm{S}(\mathrm{t})$ is strictly positive for $0<t \leq \varepsilon-\delta$, which is not possible since $S(t) \leq 0$ for all $t>0$. The same reasoning gives that $T(G) \leq T(F)-\varepsilon$ is not compatible with

$$
S^{\prime}(r)=\int_{\mathcal{R}^{2}}(\psi(x-T(F))-\psi(y-T(G)-r)) d Q(x, y) \geq 0,
$$

for all $\mathrm{r}_{\iota} 0$, and the uniform continuity of $\mathrm{T}$ in $\mathcal{U}\left(F_{0}\right)$ follows.

Some remarks: 
(a) Observe that qualitative robustness is a metric concept in the sense that, at least in principle, it depends on the metrics considered in the spaces of possible underlying and sampling distributions. A natural criterion of homogeneity might advise to use the Prokhorov metric in both spaces. However, we have used the Lévy metric in $\mathcal{F}$ due to the fact that the uniform convergence (required in the proof of Theorem 1 ) $\sup _{F} d_{L}\left(F_{n}, F\right) \longrightarrow 0$, a.s., holds for $d_{L}$. We have not found a similar result for the Prokhorov metric.

(b) The weak continuity of a statistical functional $T$ is the natural suffcient condition for the qualitative robustness of the associated sequence $\left\{T_{n}\right\}$ of estimators. Theorem 2 provides a statistical interpretation for the weak uniform continuity of $T$. Moreover, Theorem 3 points out the remarkable fact that such uniform continuity is fulfilled by broad classes of usual functionals.

\section{BoOTSTRAP CONFIDENCE BANDS FOR THE INFLUENCE CURVE}

The influence function [Hampel (1974), Hampel et al. (1987)] is already a standard tool in the current statistical theory and practice. Its definition is well-known: $T^{\prime}(F ; x)=\lim _{\epsilon \rightarrow 0^{+}}\left[T\left((1-\epsilon) F+\epsilon \delta_{x}\right)-T(F)\right] / \epsilon$, for each $F \epsilon$ $\mathcal{F}$. We assume throughout the existence and finiteness of $T^{\prime}(F ; x)$. When placed in an appropriate framework, the influence function is nothing but the integrand function arising in the expressions of the differentials used in the classical non-linear functional analysis [see, e.g., Kolmogorov and Fomin (1957)]. Its intuitive meaning and mathematical role are analogous to those of the gradient $\nabla f$ for the finite-dimensional case $f: \mathcal{R}^{n} \longrightarrow \mathcal{R}$. We will use Hadamard's concept of differentiability [for definitions, see Fernholz (1983) and Gill(1989)].

First recall that, in most cases, $T^{\prime}(F ; x)$ must be considered as a populational quantity depending upon the underlying (unknown) distribution $F$. Hence, in practice, $T^{\prime}(F ; x)$ has to be estimated from a sample $X_{1}, \ldots, X_{n}$ of $F$. Typically, this is done by replacing $F$ by $F_{n}$ and approximating the lateral derivative at zero by a quotient of finite increments. The resulting estimate is called sensitivity curve. Other useful finite-sample versions of $T^{\prime}(F ; x)$ are the empirical influence function, and the jackknife approximation [see Hampel et al. (1987), p. 92]. These approaches are free of assumptions on the functional form of $T^{\prime}(F ; x)$, but, as a matter of fact, the explicit expression of the influence function is exactly known for broad classes of estimators. For instance, under some regularity hypotheses [see Deniau et al. (1977)] the 
influence function for the M-estimators defined in Section 2 is given by

$$
T^{\prime}(F ; x)=\frac{\psi(x ; T(F))}{-\left.\int \frac{\partial}{\partial \theta} \psi(y ; \theta)\right|_{y=T(F)} d F(y)} .
$$

In the case of L-estimators, the influence function is [see Huber (1981), p. $57]$

$$
T^{\prime}(F ; x)=\int_{-\infty}^{x} h^{\prime}(y) m(F(y)) d y-\int_{-\infty}^{\infty}(1-F(y)) h^{\prime}(y) m(F(y)) d y .
$$

These important examples (as well as others which could be mentioned) suggest the following simple idea: if the functional form of the influence function $T^{\prime}(F ; x)$ is explicitly known, it can be estimated in a natural way just inserting the empirical distribution $F_{n}$ instead of the underlying $F$. Moreover, simultaneous confidence bands can be obtained by approximating the sampling distribution of

$$
D_{n}=\sup _{x} \sqrt{n}\left|T^{\prime}\left(F_{n} ; x\right)-T^{\prime}(F ; x)\right|
$$

by that of its bootstrap version

$$
D_{n}^{*}=\sup _{x} \sqrt{n}\left|T^{\prime}\left(F_{n}^{*} ; x\right)-T^{\prime}\left(F_{n} ; x\right)\right| \text {. }
$$

The validity of such an approximation is established in the following

THEOREM 4. Let $\mathcal{D}(\overline{\mathcal{R}})(\equiv \mathcal{D}[-\infty, \infty])$ denote the space of cadlag (i.e. right continuous with left-hand limits) functions on $\overline{\mathcal{R}}$, endowed with the $\|\cdot\|_{\infty}$ (essential supremum) norm. Let $T: \mathcal{F} \longrightarrow \mathcal{R}$ be a statistical functional with associated influence functional $T^{\prime}(F ; x)$. Assume that

(i) For each $F \in \mathcal{F}$, the influence function $T^{\prime}(F ;$.$) belongs to \mathcal{D}(\overline{\mathcal{R}})$ (in particular, it is bounded).

(ii) The influence functional $T^{\prime}(F ;$.$) can be extended to the vector space$ $\mathcal{G} \equiv\{\lambda(F-H): F, H \in \mathcal{F}, \lambda \in \mathcal{R}\}$ and the transformation (from $\mathcal{G}$ to $\mathcal{D}(\overline{\mathcal{R}})) H \longmapsto T^{\prime}(H ; x)$ is continuously compact differentiable.

Then, the statistic $D_{n}$ can be bootstrapped, in the sense that its bootstrap version $D_{n}^{*}$ converges weakly (a.s.) to the same limit as $D_{n}$. 
Proof. From Donsker Invariance Theorem,

$$
\sqrt{n}\left(F_{n}-F\right) \longrightarrow B^{0}(F)
$$

weakly in $\mathcal{D}(\overline{\mathcal{R}})$, where $B^{0}$ is the Brownian bridge on $[0,1]$ considered as a random element in $\mathcal{D}(\overline{\mathcal{R}})$ [see, e.g. Pollard $(1984$, p. 97)]. The $\delta$ - method (as in Gill (1989)) gives that

$$
\sqrt{n}\left(T^{\prime}\left(F_{n} ; .\right)-T^{\prime}(F ; .)\right) \longrightarrow_{w} d T^{\prime}(F ; .) B^{0}(F),
$$

where $d T^{\prime}(F ;$.$) is the Hadamard differential of T'. From the general result by$ Giné and Zinn (1990) on bootstrap of general empirical measures, we have that

$$
\sqrt{n}\left(F_{n}^{*}-F_{n}\right) \longrightarrow B^{0}(F),
$$

weakly a.s. in $\mathcal{D}(\overline{\mathcal{R}})$ and, again from Gill (1989) and hypothesis (ii), it follows that

$$
\sqrt{n}\left(T^{\prime}\left(F_{n}^{*} ; .\right)-T^{\prime}\left(F_{n} ; .\right)\right) \longrightarrow_{w} d T^{\prime}(F ; .) B^{0}(F) \text { a.s. }
$$

Now, since $\|$. $\|_{\infty}$ is continuous with respect to its own topology, by using the Continuous Mapping Theorem [see, e.g. Pollard (1984), p. 44], we conclude that $D_{n}$ and $D_{n}^{*}$ converge weakly a.s. to the same limit and the result follows.

Some remarks:

(a) This result is nothing but a direct consequence of a recent generalized version (Gill, 1989) of the classical $\delta$-method (see, e.g. Rao 1973) which is a standard tool to get asymptotic results in parametric inference. This generalization is very interesting from a mathematical point of view, although it does not seem easy to find applications in the usual problems of non-parametric functional statistics. The reason is that this technique only applies to estimates defined as restrictions of statistical functionals. In this respect, Theorem 4 can be seen as a natural example of application of this functional $\delta$-method.

(b) Note that the hypotheses of Theorem 4 are fulfilled by the influence operator of L-estimators; indeed, assuming that $h^{\prime}$ is integrable and $m$ is continuously differentiable, one can check out that the expression for this 
influence curve given above is just a composition of of continuously differentiable operators: observe that the the transformation $F \longmapsto \int_{-\infty}^{x} h^{\prime}(y) F(y) d y$ is linear and continuous and, hence, differentiable. Also, $F \mapsto m(F(\dot{)})$ is a differentiable map.

(c) In those cases where the influence function $T^{\prime}\left(F_{;}.\right)$is not bounded, Theorem 4 still holds in order to provide confidence bands on compact intervals $[-M, M]$. It would suffice to replace $\mathcal{D}(\overline{\mathcal{R}})$ by the corresponding space $\mathcal{D}[-M, M]$.

(d) Theorem 4 gives further insight on the statistical meaning of functional differentiation. Recall that the Fréchet differentiability of $T$ is a natural sufficient condition to establish the asymptotic validity of the bootstrap for $T\left(F_{n}\right)$ (see, e.g. Parr (1985)). We have proved that continuous Hadamard differentiability of $T$ allows to build bootstrap confidence bands for the influence curve.

\section{REFERENCES}

ARCONES, M.A. and GINE, E. (1992). On the bootstrap of M-estimators and other statistical functionals. Proc. IMS Bootstrap Conference, E. Lansing 1990. Wiley, New York.

BOENTE, G., FRAIMAN, R. and YOHAI, V. (1987).Qualitative robustness for stochastic processes. Ann. Statist., 15, 1293-1312.

CUEVAS, A. (1988). Qualitative robustness in abstract inference. J. Statist. Plann. Inf., 18, 277-289.

DENIAU, C., OPPENHEIM, G. and VIANO, C. (1977). Courbe d'influence et sensitivité. Asterisque 43-44, 239-252.

EFRON, B.(1982). The Jackknife, the Bootstrap, and Other Resampling Plans. CBMS Monograph 38, Society for Industrial and Applied Mathematics, Philadelphia.

FERNHOLZ, L. T. (1983). Von Mises Calculus for Statistical Functionals. Springer-Verlag, New York.

GILL, R. D. (1989). Non- and semi-parametric maximum likelihood estimators and the von Mises method (Part I) Scand. J. Statist., 16, 97-128.

GINE, E. and ZINN, J. (1990). Bootstrapping general empirical measures. Ann. Prob. 18, 851-869.

GRENANDER, U. (1981). Abstract Inference. Wiley, New York. 
HAMPEL, F.R. (1968). Contributions to the theory of robust estimation. Ph. D. Thesis. University of California, Berkeley.

HAMPEL, F.R. (1971). A general qualitative definition of robustness. Ann. Math. Stat. 42, 1887-1996.

HAMPEL, F.R. (1974). The influence curve and its role in robust estimation. J. Amer. Statist. Assoc. 69, 383-393.

HAMPEL, F.R., RONCHETTI, E.M., ROUŚSEEUW, P.J. and STAHEL, W.A. (1987). Robust Statistics. The Approach Based on Influence Functions. Wiley, New York.

HUBER, P.J. (1981). Robust Statistics. Wiley, New York.

KOLMOGOROV, A.N. and FOMIN, S.V. (1957). Elements of the Theory of Functions and Functional Analysis. Graylock, Rochester, New York.

LAMBERT, D. (1982). Qualitative robustness of tests.J. Amer. Statist. Asoc. 77, 352-357.

PARR, W. C. (1985). The bootstrap: some sample theory and conections with robustness. Stat. Prob. Letters, 3, 97-100.

POLLARD, D. (1984). Convergence of Stochastic Processes. SpringerVerlag, New York.

RAO, C.R. (1973). Linear Statistical Inference and Its Applications. Wiley, New York.

RIEDER, H. (1982). Qualitative robustness of rank tests. Ann. Statist. 10, 205-211. 